\title{
A coupled model for healthy and cancerous cells dynamics in Acute Myeloid Leukemia
}

\author{
J. L. Avila ${ }^{*}$ C. Bonnet ${ }^{*}$ H. Özbay ${ }^{* *}$ J. Clairambault ${ }^{* * *}$ \\ S. I. Niculescu ${ }^{* * * *}$ P. Hirsch ${ }^{\dagger}$ F. Delhommeau ${ }^{\ddagger}$ \\ * Inria, Equipe DISCO, LSS - SUPELEC, 3 rue Joliot Curie, 91192 \\ Gif-sur-Yvette, Cedex, France. \\ ** Dept. of Electrical and Electronics Eng., Bilkent University, Ankara, \\ 06800, Turkey. \\ *** Inria, Equipe BANG, Domaine de Voluceau, B.P. 105, 78153 Le \\ Chesney, Cedex, France, and INSERM team U 776 "Biological \\ Rhythms and Cancers", Hôpital Paul-Brousse, 14 Av. \\ Paul-Vaillant-Couturier, 94807 Villejuif, Cedex, France. \\ **** L2S (UMR CNRS 8506), CNRS-Supélec, 3 rue Joliot Curie, \\ 91192, Gif-sur-Yvette, France \\ ${ }^{\dagger}$ Sorbonne Universités, UPMC Univ Paris 6, GRC n. 07, Groupe de \\ Recherche Clinique sur les Myéloproliférations Aiguës et Chroniques \\ MyPAC, F75012 Paris, France, AP-HP, Hôpital Saint-Antoine, \\ Laboratoire d'Hématologie, F75012 Paris France, Sorbonne \\ Universités, UPMC Univ Paris 6, UMR_S 938, CDR Saint-Antoine, \\ F-75012 Paris, France and INSERM, UMR_S 938 CDR \\ Saint-Antoine, F-75012 Paris, France \\ $\ddagger$ Sorbonne Universités, UPMC Univ Paris 6, GRC n. 07, Groupe de \\ Recherche Clinique sur les Myéloproliférations Aiguës et Chroniques \\ MyPAC, F75012 Paris, France, AP-HP, Hôpital Saint-Antoine, \\ Service d'hématologie et de thérapie cellulaire, F75012 Paris France, \\ Sorbonne Universités, UPMC Univ Paris 6, UMR_S 938, CDR \\ Saint-Antoine, F-75012 Paris, France and INSERM, UMR_S 938 \\ CDR Saint-Antoine, F-75012 Paris, France
}

\begin{abstract}
In this paper we propose a coupled model for healthy and cancerous cell dynamics in Acute Myeloid Leukemia. The PDE-based model is transformed to a nonlinear distributed delay system. For an equilibrium point of interest, necessary and sufficient conditions of local asymptotic stability are given. Simulation examples are given to illustrate the results.
\end{abstract}

Keywords: Modelling, PDE, Delay, Medical applications, nonlinear models.

\section{INTRODUCTION}

The process of blood cell production is called hematopoiesis Starting in the bone marrow by hematopoiteic stem cells (HSCs), the blood-forming system is an example of a multistagesytem. At the first level, HSCs can proliferate, self renew and differentiate into multiple lineages. The process of cell division, called proliferation or cell cycle, consists of four phases: $G_{1}, S, G_{2}$ and $M$. At the end of the $M$ phase cell division occurs and two types of daughter cells may be produced: either with the same maturity as the parent or with a higher level of maturity through a differentiation process. Finally, several stages down, fully differentiated cells are released in blood circulation. One of the first mathematical models on hematopoiesis was proposed by Mackey (1978). This model consists of a system of differential equations, describing haematopoietic stem cell dynamics, considering a rest (or quiescent)

* This work was supported by the DIGITEO Project ALMA partly funded by the Région Île-de-France, France phase and a proliferative phase during the cell division cycle. More recent studies of various dynamical models of hematopoiesis have been proposed and studied in the literature, see e.g. Adimy et al. (2008), Dingli and Pacheco (2010), Foley and Mackey (2009), Niculescu et al. (2010).

AML combines at least two molecular events: a blockade of the maturation and differentiation leading to the accumulation of immature myeloid cells, and an advantage of proliferation leading to the flooding of bone marrow by a large number of immature cells. In Avila et al. (2012), a system of delay-differential equations, inspired by the model of Adimy et al. (2008), with discrete maturity structure has been proposed as a model for the AML. The model takes into account the differentiation blockade that is constantly observed in AML. From the biological and medical point of view, the healthy situation may be defined by stable equilibrium representing extinction of cancerous cells with positive value for healthy cells. The aim of such a modelling is to yield conditions that make 
biological and medical sense ensuring a disease-free state for hematopoiesis in the bone marrow. This will further give goals to be pursued in the hematopoiesis of AML. For the equilibrium and stability analysis (linear and nonlinear system) of this model see Ozbay et al. (2012), Adimy et al. (2008) and the references given there. A unique model mixing as a sum of healthy and cancerous cells is obviously unidentifiable. However, some biological experiments performed on fresh blood samples from leukemic patients makes it possible to identify parameters of such a cancerous cell dynamics model.

In this paper, we consider a mathematical model based on the interconnection of the populations of healthy and cancerous cells. For the modelling of the healthy cells, we consider the mathematical model introduced in Adimy et al. (2008). The dynamical behavior of the cancerous cells is based on the model studied in Avila et al. (2012) which models the effect of AML cells as follows. Self-renewal, i.e. the fate of cells that do not change their maturity for each fixed maturity stage is separated into two parts: slow dynamics for those cells that go to the classical quiescent phase $G_{0}$ and fast dynamics for those that come back very fast to the proliferative compartment through a transient phase $\tilde{G}_{0}$. It is important to take these fast dynamics into account because of the existence of anti-proliferative drugs (inhibitors of Flt-3 receptors) that act specifically on this fast cell renewal dynamics.

For both populations of healthy and cancerous cells, we consider that its proliferating cells are separated into three phases: $G_{1}, S, G_{2} M$. Biological experiments performed at St Antoine Hospital in Paris, France, based on flow cytometry and aiming at identifying parameters of the model do not allow us to distinguish between $G_{2}$ and $M$ phases which explains why we aggregate these two phases in our model. The coupling between the two populations healthy and cancerous cells, is modelled by the feedback function $\beta$ that takes into account the sum of both populations of resting cells. In this paper we only consider one stage of maturity.

The structure of this paper is as follows. Section 2 provides a detailed exposition of the partial differential equations that govern the dynamics of the healthy and cancerous cells. It is also shown how to derive a nonlinear distributed delay model, by using the method of characteristics. Section 3 is devoted first to the study of the existence of nonnegative equilibrium points of the nonlinear delay model. Then, we obtain a linearized model. Finally, a stability analysis of the linear system around a suitable equilibrium point is discussed. A detailed academic example is presented in Section 5. Finally, in Section 6, some concluding remarks are outlined.

\section{MATHEMATICAL MODEL OF A COUPLED POPULATION OF HEALTHY AND CANCEROUS CELLS}

\subsection{Partial Differential Equation Model}

Let us consider two populations of cells representing the healthy and the cancerous cells. We denote by $p(t, a)$, $l(t, a), n(t, a)$ and $r(t, a)$ the cell populations of cancerous immature cells of the $G_{1}, S, G_{2} M$ and $G_{0}$ phases respec- tively, with age $a \geq 0$ at time $t \geq 0$. The fast-renewal effect of AML cells is represented by a new transient phase, located at the end of the phase $M$, called $\tilde{G}_{0}$ and its population is denoted by $\tilde{r}(t, a)$. The dynamical behavior of the cancerous cells is represented by the following system of transport equations structured in age $a$ :

$$
\begin{aligned}
& \left(\partial_{t} p+\partial_{a} p=-\left(\gamma^{1}+g^{p}(a)\right) p, \quad 0<a<\tau^{1}, t>0,\right. \\
& \partial_{t} l+\partial_{a} l=-\left(\gamma^{2}+g^{l}(a)\right) l, \quad 0<a<\tau^{2}, t>0, \\
& \partial_{t} n+\partial_{a} n=-\left(\gamma^{3}+g^{n}(a)\right) n, \quad 0<a<\tau^{3}, t>0, \\
& \left\{\partial_{t} r+\partial_{a} r=-(\delta+\beta(z(t))) r, \quad a>0, t>0,\right. \\
& \partial_{t} \tilde{r}+\partial_{a} \tilde{r}=-\delta \tilde{r} \\
& +\beta\left(\int_{0}^{+\infty} \tilde{r}(t, a) d a\right) \\
& a>0, t>0 \text {, }
\end{aligned}
$$

On the other hand, $\bar{p}(t, a), \bar{l}(t, a), \bar{n}(t, a)$ and $\bar{r}(t, a)$ represent the cell populations of the $G_{1}, S, G_{2} M$ and $G_{0}$ phases respectively, of healthy cells, with age $a \geq 0$ at time $t \geq 0$. The dynamical behavior of the healthy cells is represented by the following system of transport equations structured in age $a$ :

$$
\begin{cases}\partial_{t} \bar{p}+\partial_{a} \bar{p}=-\left(\bar{\gamma}^{1}+\bar{g}^{\bar{p}}(a)\right) \bar{p}, & 0<a<\bar{\tau}^{1}, t>0, \\ \partial_{t} \bar{l}+\partial_{a} \bar{l}=-\left(\bar{\gamma}^{2}+\bar{g}^{\bar{l}}(a)\right) \bar{l}, & 0<a<\bar{\tau}^{2}, t>0, \\ \partial_{t} \bar{n}+\partial_{a} \bar{n}=-\left(\bar{\gamma}^{3}+\bar{g}^{\bar{n}}(a)\right) \bar{n}, & 0<a<\bar{\tau}^{3}, t>0, \\ \partial_{t} \bar{r}+\partial_{a} \bar{r}=-(\bar{\delta}+\bar{\beta}(z(t))) r, & a>0, t>0,\end{cases}
$$

The functions and variables needed to read (1) and (2) have the following properties and biological meaning: the death rates $\delta$ and $\bar{\delta}$ satisfy $\delta>0$ and $\bar{\delta}>0$; the functions $\beta$ and $\bar{\beta}$ are re-introduction functions from the resting subpopulation into the proliferative subpopulation of cancerous and healthy cells, respectively; $\gamma^{1}, \gamma^{2}, \gamma^{3}$ and $\bar{\gamma}^{1}, \bar{\gamma}^{2}, \bar{\gamma}^{3}$ are constant death rates in the $G_{1}, S, G_{2} M$ phases of cancerous and healthy cells, respectively; the amount of time spent in the $G_{1}, S, G_{2} M$ phases are $\tau^{1}$, $\tau^{2}, \tau^{3}$ for the healthy cells and $\bar{\tau}^{1}, \bar{\tau}^{2}, \bar{\tau}^{3}$ for the cancerous cells; and, the division rates of the phases $G_{1}, S, G_{2} M$ are functions depending on $a$, denoted by $g^{p}, g^{l}, g^{n}$ for the healthy cells and $\bar{g}^{\bar{p}}, \bar{g}^{\bar{l}}, \bar{g}^{\bar{n}}$ for the cancerous cells.

For the cancerous cells,

$$
x(t):=\int_{0}^{+\infty} r(t, a) d a
$$

and

$$
\tilde{x}(t):=\int_{0}^{+\infty} \tilde{r}(t, a) d a
$$

stand for the total population of resting and fast selfrenewing cells at the time $t$, respectively. Otherwise, the total population of resting healthy cells is denoted by

$$
\bar{x}(t):=\int_{0}^{+\infty} \bar{r}(t, a) d a .
$$

The healthy and cancerous cells are interconnected by means of the common feedback of resting cells $z(t)=$ $x(t)+\bar{x}(t)$ on the functions $\beta$ and $\bar{\beta}$. 


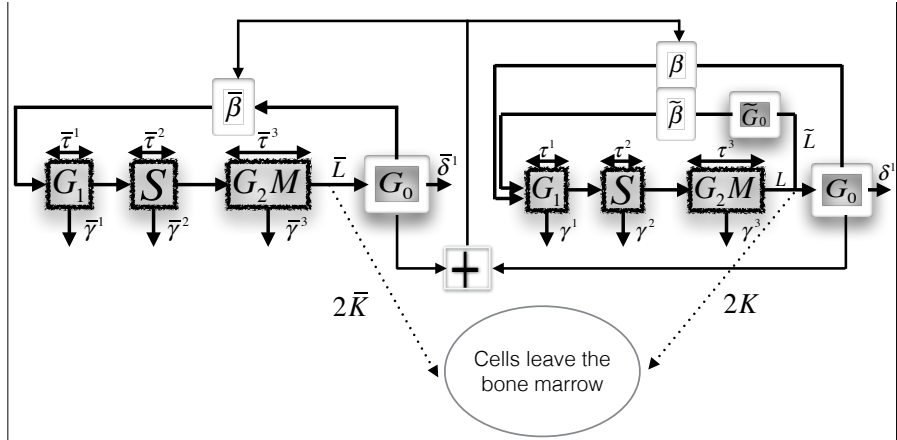

Fig. 1. Interconnected model of healthy cells (left) and cancerous cells (right)

Boundary conditions associated with (1) and (2) are given by:

$$
\left\{\begin{array}{l}
p(t, a=0)=\beta(x(t)+x \overline{(t)}) x(t)+\tilde{\beta}(\tilde{x}(t)) \tilde{x}(t) \\
l(t, a=0)=\int_{0}^{\tau^{1}} g^{p}(a) p(t, a) d a \\
n(t, a=0)=\int_{0}^{\tau^{2}} g^{l}(a) l(t, a) d a \\
r(t, a=0)=L \int_{0}^{\tau^{4}} g^{n}(a) n(t, a) d a \\
\tilde{r}(t, a=0)=\tilde{L} \int_{0}^{\tau^{4}} g^{n}(a) n(t, a) d a
\end{array}\right.
$$

for the cancerous cells; and

$$
\left\{\begin{array}{l}
\bar{p}(t, a=0)=\bar{\beta}(x(t)+\bar{x}(t)) \bar{x}(t) \\
\bar{l}(t, a=0)=\int_{0}^{\bar{\tau}^{1}} g^{\bar{p}}(a) \bar{p}(t, a) d a, \\
\bar{n}(t, a=0)=\int_{0}^{\bar{\tau}^{2}} g^{\bar{l}}(a) \bar{l}(t, a) d a, \\
\bar{r}(t, a=0)=L \int_{0}^{\bar{\tau}^{4}} g^{\bar{n}}(a) \bar{n}(t, a) d a
\end{array}\right.
$$

for the healthy cells; we define $L=2 \sigma(1-K)$, $\tilde{L}=2(1-\sigma)(1-K)$ and $\bar{L}=2(1-\bar{K})$. The constants $K$, and $\bar{K}$ represent the probability of differentiation of daughter cells so that $0<K<1$ and $0<\bar{K}<1$. The constant $\sigma$ represents the probability of fast self-renewal $0<\sigma<1$.

The initial age-distribution of the populations of (1) are nonnegative age-dependent functions and they are assumed to be known: $p(t=0, a)=p^{0}(a), l(t=0, a)=$ $l^{0}(a), n(t=0, a)=n^{0}(a), r(t=0, a)=r^{0}(a)$, $\tilde{r}(t=0, a)=\tilde{r}^{0}(a), \bar{p}(t=0, a)=p^{0}(a), \bar{l}(t=0, a)=$ $\bar{l}^{0}(a), \bar{n}(t=0, a)=\bar{n}^{0}(a)$,

and $\bar{r}(t=0, a)=\bar{r}^{0}(a)$.

The following assumptions complete the mathematical model:

(1) The division rates $g^{p}, g^{l}, g^{n}, g^{\bar{p}}, g^{\bar{l}}$ and $g^{\bar{n}}$ are continuous functions such that $\int_{0}^{\tau^{1}} g^{p}(a) d a=+\infty, \int_{0}^{\tau^{2}} g^{l}(a) d a=+\infty$, $\int_{0}^{\tau^{3}} g^{n}(a) d a=+\infty, \quad \int_{0}^{\bar{\tau}^{1}} g^{\bar{p}}(a) d a=+\infty$, $\int_{0}^{\bar{\tau}^{2}} g^{\bar{l}}(a) d a=+\infty$ and $\int_{0}^{\bar{\tau}^{3}} g^{\bar{n}}(a) d a=+\infty$.
(2) $\lim _{a \rightarrow+\infty} r(t, a)=0, \lim _{a \rightarrow+\infty} \tilde{r}(t, a)=0$ and $\lim _{a \rightarrow+\infty} \bar{r}(t, a)=0$.

(3) The re-introduction functions $\beta, \tilde{\beta}$ and $\tilde{\beta}$ are differentiable, non-negative and uniformly decreasing functions.

In this paper, we are interested in functions $\beta, \tilde{\beta}$ and $\bar{\beta}$ of the form

$\beta(x)=\frac{\beta(0)}{1+b x^{N}}, \tilde{\beta}(\tilde{x})=\frac{\tilde{\beta}(0)}{1+\tilde{b} \tilde{x}^{\tilde{N}}}$ and $\bar{\beta}(\bar{x})=\frac{\bar{\beta}(0)}{1+\bar{b} \bar{x}^{\bar{N}}}$

where $N, \tilde{N}$ and $\tilde{N}$ are integers greater or equal to $2 ; b>0$, $\tilde{b}>0, \bar{b}>0$ and $\tilde{b} \ll 1$. These functions are known as Hill functions.

Next, we shall give a system of ordinary differential distributed delayed equations obtained from the PDE's (1) and (2) .

\subsection{Distributed Delay Differential Equation Model}

By using the method of characteristics and applying the same rationale as in Avila et al. (2012) we obtain the following systems of distributed delay differential equations

$$
\begin{aligned}
\dot{x}(t)= & -(\delta+\beta(x(t)+\bar{x}(t))) x(t) \\
& +L\left(h^{3} * h^{2} * h^{1} * \omega\right)(t) \\
\dot{\tilde{x}}(t)= & -\tilde{\beta}(\tilde{x}(t)) \tilde{x}(t)+\tilde{L}\left(h^{3} * h^{2} * h^{1} * \omega\right)(t) \\
\dot{\bar{x}}(t)= & -(\bar{\delta}+\bar{\beta}(x(t)+\bar{x}(t))) \bar{x}(t) \\
& +\bar{L}\left(\bar{h}^{3} * \bar{h}^{2} * \bar{h}^{1} * \bar{\omega}\right)(t)
\end{aligned}
$$

where $\omega(t):=\beta(x(t)+\bar{x}(t)) x(t)+\tilde{\beta}(\tilde{x}(t)) \tilde{x}(t)$, $\bar{\omega}(t):=\bar{\beta}(x(t)+\bar{x}(t)) \bar{x}(t), h^{1}(t):=f^{p}(t) e^{-\gamma^{1} t}$, $h^{2}(t):=f^{l}(t) e^{-\gamma^{2} t}, h^{3}(t):=f^{n}(t) e^{-\gamma^{3} t}$, $\bar{h}^{1}(t):=f^{\bar{p}}(t) e^{-\bar{\gamma}^{1} t}, \bar{h}^{2}(t):=f^{\bar{l}}(t) e^{-\bar{\gamma}^{2} t}$ and $\bar{h}^{3}(t):=f^{\bar{n}}(t) e^{-\bar{\gamma}^{3} t}$. The functions $f^{p}, f^{l}, f^{n}, f^{\bar{p}}, f^{\bar{l}}$ and $f^{\bar{n}}$ are density functions on the intervals $\left[0, \tau^{1}\right],\left[0, \tau^{2}\right]$, $\left[0, \tau^{3}\right],\left[0, \bar{\tau}^{1}\right],\left[0, \bar{\tau}^{2}\right]$ and $\left[0, \bar{\tau}^{3}\right]$. The symbol $*$ stands for the usual convolution operator.

As in Avila et al. (2012), we will consider the following general form for the division rates $h^{i}$ and $\bar{h}^{i}$ :

and

$$
h^{i}(t)=\frac{m^{i}}{e^{m^{i} \tau^{i}}-1} e^{\left(m^{i}-\gamma^{i}\right) t} \text { for } 0 \leq t \leq \tau^{i}
$$

$$
\bar{h}^{i}(t)=\frac{\bar{m}^{i}}{e^{\bar{m}^{i} \bar{\tau}^{i}}-1} e^{\left(\bar{m}^{i}-\bar{\gamma}^{i}\right) t} \text { for } 0 \leq t \leq \tau^{i} .
$$

This gives

$$
H^{i}(s):=\int_{0}^{\tau^{i}} h^{i}(t) e^{-s t} d t=q^{i}\left(\frac{1-e^{-\tau^{i}\left(s-r^{i}\right)}}{s-r^{i}}\right)
$$

and

$$
\bar{H}^{i}(s):=\int_{0}^{\bar{\tau}^{i}} \bar{h}^{i}(t) e^{-s t} d t=\bar{q}^{i}\left(\frac{1-e^{-\bar{\tau}^{i}\left(s-\bar{r}^{i}\right)}}{s-\bar{r}^{i}}\right)
$$

with $q^{i}:=\frac{m^{i}}{e^{m^{i} \tau^{i}}-1}, \bar{q}^{i}:=\frac{\bar{m}^{i}}{e^{\bar{m}^{i} \bar{\tau}^{i}}-1}, r^{i}:=m^{i}-\gamma^{i}$ and $\bar{r}^{i}:=\bar{m}^{i}-\bar{\gamma}^{i}$

In the next section, we will study some qualitative properties of the nonlinear system derived above. 


\section{MODEL LINEARIZATION AND STABILITY}

\subsection{Nonnegative equilibrium points}

Let us denote by $x^{e}, \tilde{x}^{e}$ and $\bar{x}^{e}$ the equilibrium points of (4)-(6). Clearly, the origin is an equilibrium of the nonlinear system.

Equating to zero the right hand side of (4)-(6), it may be concluded that on the equilibrium the re-introduction functions satisfy the following relations:

$$
\begin{aligned}
\beta\left(x^{e}+\bar{x}^{e}\right) & =-\frac{(1-\tilde{L} H(0)) \delta}{1-(L+\tilde{L}) H(0)} \\
\tilde{\beta}\left(\tilde{x}^{e}\right) & =-\frac{\delta \tilde{L} H(0)}{(1-(L+\tilde{L}) H(0))}\left(\frac{x^{e}}{\tilde{x}^{e}}\right) \\
\bar{\beta}\left(x^{e}+\bar{x}^{e}\right) & =-\frac{\bar{\delta}}{1-\bar{L} \bar{H}(0)}
\end{aligned}
$$

Note that on the equilibrium: 1 ) the right-hand side of (9)(6) must be positive due to the non-negativeness of the re-introduction functions $\beta, \tilde{\beta}$ and $\bar{\beta} ; 2$ ) the decreasing property of the functions $\beta$ and $\bar{\beta}$ implies that $\beta(0)>$ $\beta\left(x^{e}+\bar{x}^{e}\right)$ and $\bar{\beta}(0)>\bar{\beta}\left(x^{e}+\bar{x}^{e}\right)$. Summarizing, we have thus proved that the following conditions are necessary for the existence of positive equilibrium points:

$$
\begin{gathered}
\beta(0)>-\frac{(1-\tilde{L} H(0)) \delta}{1-(L+\tilde{L}) H(0)} \delta \\
\bar{\beta}(0)>-\frac{\bar{\delta}}{1-\bar{L} \bar{H}(0)} \\
1-L \bar{H}(0)<0 \\
1-(L+\tilde{L}) H(0)<0 \\
1-\tilde{L} H(0)>0
\end{gathered}
$$

Now, we discuss under which conditions the following two cases of equilibrium points have solutions: 1) $x^{e}=0$, $\left.\tilde{x}^{e}=0, \bar{x}^{e}>0 ; 2\right) x^{e}>0, \tilde{x}^{e}>0$ and $\bar{x}^{e}>0$.

First, when $x^{e}=0, \tilde{x}^{e}=0$, the conditions (13) and (14) are necessary and sufficient for the existence of a unique equilibrium point $\bar{x}^{e}>0$, see Adimy et al. (2008).

Finally, when we are interested in strictly positive equilibrium points the following relation must be fulfilled by $x^{e}$ and $\bar{x}^{e}$ on the assumption that $\beta, \tilde{\beta}$ and $\bar{\beta}$ are Hill functions:

$$
x^{e}+\bar{x}^{e}= \begin{cases}\left(\frac{(\beta(0)+c-1) \bar{b}}{(\bar{\beta}(0)+\bar{c}-1) b}\right)^{(1 /(N-\bar{N}))}, & \text { if } N>\bar{N} \\ \left(\frac{(\bar{\beta}(0)+\bar{c}-1) b}{(\beta(0)+c-1) \bar{b}}\right)^{(1 /(\bar{N}-N))}, & \text { if } N<\bar{N}\end{cases}
$$

and when $N=\bar{N}$

$x^{e}+\bar{x}^{e}=\left(\frac{\beta(0)+c-1}{b}\right)^{\left(1 / N_{1}\right)}=\left(\frac{\bar{\beta}(0)+\bar{c}-1}{\bar{b}}\right)^{(1 / \bar{N})}$

where $c=-\frac{(1-\tilde{L} H(0)) \delta}{1-(L+\tilde{L}) H(0)}$ and $\bar{c}=-\frac{\bar{\delta}}{1-\bar{L} \bar{H}(0)}$.

The perturbation of the equilibrium point $x^{e}=0, \tilde{x}^{e}=0$ and $\bar{x}^{e}>0$ may provoke the birth of cancerous cells and therefore we analyze its stability conditions in the next section.

\subsection{Stability analysis}

Consider the following perturbed trajectories: $X(t):=$ $x(t)-x^{e}, \tilde{X}(t):=\tilde{x}(t)-\tilde{x}^{e}$ and $\bar{X}(t):=\bar{x}(t)-\bar{x}^{e}$. Taking the time derivative of the perturbed trajectories, we obtain that the linearized model around the equilibrium point $x^{e}, \tilde{x}^{e}$ and $\bar{x}^{e}$ is represented by:

$$
\begin{aligned}
\dot{X}(t)= & -(\delta+\mu) X(t)-c_{12} \bar{X}(t) \\
& +L\left(h^{3} * h^{2} * h^{1} * W\right)(t) \\
\dot{\tilde{X}}(t)= & -\tilde{\mu} \tilde{X}(t)+\tilde{L}\left(h^{3} * h^{2} * h^{1} * W\right)(t) \\
\dot{\bar{X}}(t)= & -(\bar{\delta}+\bar{\mu}) \bar{X}(t)-c_{15} X_{1}(t) \\
& +\bar{L}\left(\bar{h}^{3} * \bar{h}^{2} * \bar{h}^{2} * \bar{W}\right)(t)
\end{aligned}
$$

where $W_{1}(t):=\mu X_{1}(t)+c_{12} \bar{X}_{1}(t)+\tilde{\mu} \tilde{X}_{1}(t)$,

$\bar{W}_{1}(t):=\bar{\mu} \bar{X}_{1}(t)+c_{15} X_{1}(t), \mu=\left.\frac{d}{d x}(\beta(x+\bar{x}) x)\right|_{\substack{x=x^{e} \\ \bar{x}=\bar{x}^{e}}}$,

$c_{12}=\left.\frac{d}{d \bar{x}}(\beta(x+\bar{x}) x)\right|_{\substack{x=x^{e} \\ \bar{x}=\bar{x} e^{e}}}, \tilde{\mu}=\left.\frac{d}{d \tilde{x}}(\tilde{\beta}(\tilde{x}) \tilde{x})\right|_{\tilde{x}=\tilde{x}^{e}}$,

$\bar{\mu}=\left.\frac{d}{d \bar{x}}(\bar{\beta}(x+\bar{x}) \bar{x})\right|_{\substack{x=x^{e} \\ \bar{x}=\bar{x}^{e}}} ^{\bar{x}=\bar{x}}$

and $c_{15}=\left.\frac{d}{d x_{1}}(\bar{\beta}(x+\bar{x}) \bar{x})\right|_{\substack{x=x^{e} \\ \bar{x}=\bar{x}}}$.

Taking the Laplace transform of the linearized model (9)-(6) and setting $H(s)=H^{1}(s) \cdot H^{2}(s) \cdot H^{3}(s)$ and $\bar{H}(s)=\bar{H}^{1}(s) \cdot \bar{H}^{2}(s) \cdot \bar{H}^{3}(s)$, we have that the linear system (18)-(20) is stable if and only if $1 / \operatorname{det}(A(s))$ is stable, where

$$
A(s)=\left[\begin{array}{ccc}
a_{11}(s) & a_{12}(s) & a_{13}(s) \\
a_{21}(s) & a_{22}(s) & a_{23}(s) \\
a_{31}(s) & 0 & a_{33}(s)
\end{array}\right]
$$

$a_{11}(s):=s+\delta+\mu-\mu L H(s), a_{12}(s):=-\tilde{\mu} L H(s)$, $a_{13}(s):=c_{12}-c_{12} L H(s), a_{21}(s):=-\mu \tilde{L} H(s)$, $a_{22}(s):=s+\tilde{\mu}-\tilde{\mu} \tilde{L} H(s), a_{23}(s):=-c_{12} \tilde{L} H(s)$, $a_{31}(s)=c_{15}-c_{15} \bar{L} \bar{H}(s)$ and $a_{33}(s):=s+\bar{\delta}_{1}+\bar{\mu}-\bar{\mu} \bar{L}(s)$.

Consequently, the stability of the linearized model is determined by the roots of

$$
\begin{aligned}
a_{33}(s) & \left(a_{11}(s) a_{22}(s)-a_{12}(s) a_{21}(s)\right) \\
& -a_{31}(s)\left(a_{12}(s) a_{23}(s)-a_{13}(s) a_{22}(s)\right)
\end{aligned}
$$


Now, we will show how to obtain bounds on $H$ and $\bar{H}$ such that the stability of the equilibrium point $x=0, \tilde{x}=0$ and $\bar{x}^{e}>0$ is guaranteed.

Proposition 1. Suppose that $\bar{\delta}+\bar{\mu}>0, \delta+\mu>0$, $\tilde{\mu}>0$, then the linearized system (18)-(20) around the equilibrium point $x=0, \tilde{x}=0$ and $\bar{x}^{e}>0$ is stable if and only if

$$
H(0)<\frac{\delta+\mu}{2(1-K)(|\mu|+(1-\sigma) \delta)}
$$

and

$$
\bar{H}(0)<\frac{\bar{\delta}+\bar{\mu}}{2(1-\bar{K})|\bar{\mu}|} .
$$

Then the system (18)-(20) is asymptotically stable. In particular, the nonlinear system (4)-(6) is locally asymptotically stable.

Proof. Since we are interested in studying an equilibrium point of the form $x^{e}=0, \tilde{x}^{e}=0$ and $\bar{x}^{e}>0$, we can see that at such an equilibrium $c_{12}=0$. This gives $a_{13}(s)=0$ and $a_{23}(s)=0$. Thus, the stability of the equilibrium point $x^{e}=0, \tilde{x}^{e}=0$ and $\bar{x}^{e}>0$ depends on the roots of

$$
a_{33}(s)\left(a_{11}(s) a_{22}(s)-a_{12}(s) a_{21}(s)\right)
$$

which is due to the fact that $A(s)$ is a block lower triangular matrix. From this, it may be concluded that stability of the linearized system is determined by the location of the roots of

$$
s+\bar{\delta}+\bar{\mu}-\bar{\mu} \bar{L} \bar{H}(s)
$$

and

$$
(s+\delta+\mu)(s+\tilde{\mu})-(\delta \tilde{L}+(L+\tilde{L}) \mu) \tilde{\mu} H(s) .
$$

We can rewrite (25) and (26) as

$$
(s+\bar{\delta}+\bar{\mu})\left(1-\frac{\bar{\mu} \bar{L} \bar{H}(s)}{s+\bar{\delta}+\bar{\mu}}\right)
$$

and

$$
(s+\delta+\mu)(s+\tilde{\mu})\left(1-\left(\frac{L \mu}{s+\delta+\mu}+\frac{\tilde{L} \tilde{\mu}}{s+\tilde{m u}}\right) H(s)\right) .
$$

From the fact that $\|H\|_{\infty}=H(0),\|\bar{H}\|_{\infty}=\bar{H}(0)$ and that $\frac{\bar{\mu} \bar{L} \bar{H}(s)}{s+\bar{\delta}+\bar{\mu}}$ and $\frac{L \mu}{s+\delta+\mu}+\frac{\tilde{L} \tilde{\mu}}{s+\tilde{m u}}$ are low pass filters whose $H_{\infty}$ norms are attained at $s=0$. Then, by the Nyquist stability criterion (due to positive feedback, i.e. sign in the characteristic equation), we see that the roots of (24) are in the open left half plane if and only if (22) and (23) hold.

\section{NUMERICAL EXAMPLES AND SIMULATION

\begin{tabular}{|c|c|c|c|c|c|c|c|c|c|}
\hline \multicolumn{2}{|c|}{$\beta(0)$} & $\tilde{\beta}(0)$ & $\bar{\beta}(0)$ & $b$ & $\tilde{b}$ & $\bar{b}$ & $N$ & $\tilde{N}$ & $\bar{N}$ \\
\hline \multicolumn{2}{|c|}{2} & 1 & 15 & 1 & 0.1 & 1 & 2 & 2 & 3 \\
\hline$m^{1}$ & $m$ & $m^{3}$ & $\tau^{1}$ & $\tau^{2}$ & $\overline{\tau^{3}}$ & $\gamma$ & & $\gamma^{2}$ & $\gamma^{3}$ \\
\hline 3 & 1 & 2 & 0.3 & 0.1 & 0.3 & & & 0.01 & 0.02 \\
\hline $\bar{m}^{1}$ & $\bar{m}$ & $\bar{m}^{3}$ & $\bar{\tau}^{1}$ & $\bar{\tau}^{2}$ & $\bar{\tau}^{3}$ & $\bar{\gamma}$ & & $\bar{\gamma}^{2}$ & $\bar{\gamma}^{3}$ \\
\hline 3 & 1 & 2 & 1.3 & 0.1 & 0.2 & & & 0.01 & 0.02 \\
\hline
\end{tabular} RESULTS}

First we present a locally stable equilibrium case, where $\delta=2, \bar{\delta}=10.3, K=0.1$ and $\bar{K}=0.2, \sigma=0.9$ and the other parameters as indicated in Table 1 . The resulting equilibrium point is $\bar{x}^{e}=0.60, x^{e}=\tilde{x}^{e}=0$; with the parameters $\mu=1.52, \tilde{\mu}=1$ and $\bar{\mu}=7.01$.
Time domain simulation, performed in Matlab Simulink, shows that with the initial conditions

$$
\begin{aligned}
& x(\tau)=0.5 \quad \text { for all }-0.3 \leq \tau<0 \\
& \bar{x}(\tau)=0.2 \quad \text { for all }-1.3 \leq \tau<0
\end{aligned}
$$

$\tilde{x}(\tau)=0$, for all $\tau<0, x(0)=1, \tilde{x}(0)=0.38$ and $\bar{x}(\tau)=1.05$ the states converge to the equilibrium points, see Figure 2. Indeed it can be verified that with the parameters in Table 1 the local stability conditions stated in (13) are satisfied: $\bar{\delta}+\bar{\mu}=27.31, \delta+\mu=3.52, \tilde{\mu}=1$,

$$
H(0)=0.9922<\frac{\delta+\mu}{2(1-K)(|\mu|+(1-\sigma) \delta)}=1.1364
$$

and

$$
\bar{H}(0)=0.9681<\frac{\bar{\delta}+\bar{\mu}}{2(1-\bar{K})|\bar{\mu}|}=1.2468
$$

To recap, in this numerical study we see that both populations of cancerous cells $x$ and $\tilde{x}$ decay to zero while healthy cells population converges to a positive stable steady-state.

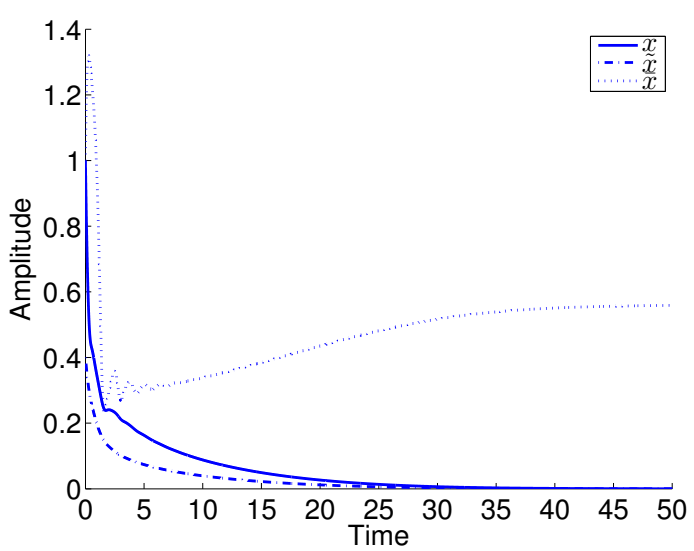

Fig. 2. Trajectories of the states $x, \tilde{x}$ and $\bar{x}$ : stable case

To complement the above numerical study we also illustrate the cell population dynamics in a locally unstable case. Further simulations show that decreasing $\sigma$ and fixing the remaining parameters as in the previous example the local stability condition is no longer satisfied. For example, when $\sigma=0.3$ the state $\tilde{x}$ is far from its equilibrium value, see Figure 3. 


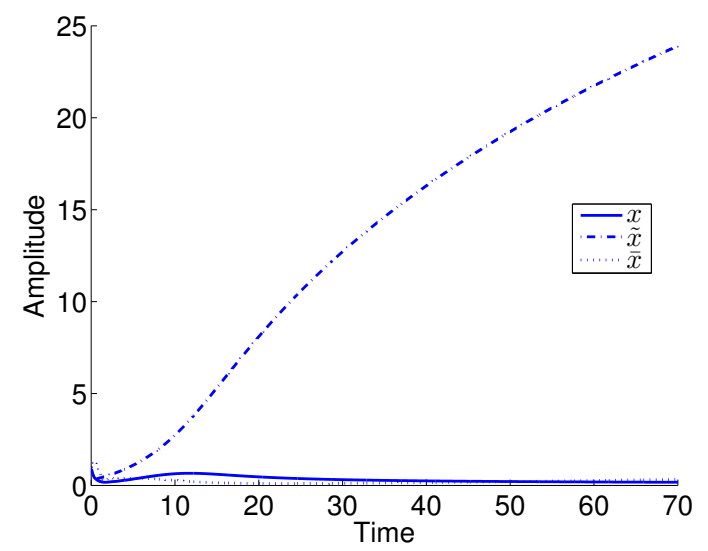

Fig. 3. Trajectories of the states $x, \tilde{x}$ and $\bar{x}$ : unstable case

\section{CONCLUSIONS}

In this paper, we have proposed a interconnected model of healthy cells and cancerous cells of AML. The PDEs governing the dynamics of the cells are based on the models of (Adimy et al. (2008)), healthy cells, and (Avila et al. (2012)), cancerous cells. Due to technical difficulties with the identification of the parameters of the phases $G_{2}$ and $M$, we modelled these two phases as a single one named $G_{2} M$ for both models of healthy and cancerous cells. Future work will consider several maturation stages in the model, both for cancerous and healthy cells.

\section{REFERENCES}

Adimy, M., Crauste, F., and El Abdllaoui, A. (2008). Discrete maturity-structured model of cell differentiation with applications to acute myelogenous leukemia. $J$. Biological Systems, 16, No. 3, 395-424.

Adimy, M., Crauste, F., and Marquet C. (2010). Asymptotic behavior and stability switch for a matureimmature model of cell differentiation. Nonlinear Analysis: Real World Applications, 11, No. 4, 2913-2929.

Avila, J.L., Bonnet, C., Clairambault, J., Özbay, H., Niculescu, S-I., Merhi, F., Tang, R., Marie, J.P., A new model of cell dynamics in Acute Myeloid Leukemia involving distributed delays. Proc. of 10th IFAC Workshop on Time Delay Systems, Boston, USA, June 2012, pp. 55-60.

Dingli, D., and Pacheco, J. M. (2010). Modeling the architecture and dynamics of hematopoiesis. Wiley Interdisciplinary Reviews: Systems Biology and Medicine, 2, No. 2, 235-244.

Foley, C., and Mackey, M.C. (2009). Dynamic hematological disease: a review. J. Mathematical Biology, 58, No. 12, 285-322.

Mackey, M. C. (1978). Unified hypothesis for the origin of aplastic anaemia and periodic hematopoiesis. Blood, 51, No. 5, 941-956.

Niculescu, S-I., Kim, P. S., Gu, K., Lee, P.P., and Levy, D. (2010). Stability crossing boundaries of delay systems modeling immune dynamics in leukemia. Discrete and Continuous Dynamical Systems Series B, 13, No. 1, 129156.

Özbay, H., Bonnet, C.,Benjelloun, H., and Clairambault, J. (2012). Stability Analysis of Cell Dynamics in
Leukemia. Mathematical Modelling of Natural Phenomena, 7, No. 1, pp. 203-234.

Özbay, H., Benjelloun, H., Bonnet, C., Clairambault, J. (2010). Stability conditions for a system modeling cell dynamics in leukemia. Preprints of IFAC Workshop on Time Delay Systems, TDS2010, Prague, Czech Republic, June 2010.

Perthame, B. (2007). Transport equations in biology. Frontiers in Mathematics, Birkhäuser Verlag.

Rowe, J. (2008). Why is clinical progress in acute myelogenous leukemia so slow? Best Practice \& Research Clinical Haematology, vol. 21, No. 1, pp. 1-3. 